\title{
Basic life support training for the adult lay population. A systematic review
}

\author{
Lourdes Luque-López ${ }^{1, *}$, Jesús Molina-Mula ${ }^{1}$
}

${ }^{1}$ Department of Nursing and

Physiotherapy, University of the Balearic

Islands, Palma de Mallorca, Spain

\section{*Correspondence}

lourdes.luque.hse@gmail.com

(Lourdes Luque-López)

\begin{abstract}
Cardiopulmonary resuscitation (CPR) training is an influential method for improving the perception of resuscitation maneuvers, user willingness to carry it out, and also coordination of the people performing it. The main objective of the present systematic review is to evaluate the effects of basic CPR training program and Semi-Automatic External Defibrillation (AED) use on acquiring and retaining practical skills of lay adults. The included researches involved adult participants of 18 years old and over without previous CPR official training. In addition, the studies containing any training program, without a specified training method of CPR and AED use, were considered. A qualitative analysis was conducted to determine the most efficient B-CPR and AED use training program for lay adults regarding to acquisition and retention of practical skills. All randomized experimental trials followed a sequence of assigning participants to different categories. This corresponds to distinct interventions which in turn involved various CPR and AED training types or different durations of training between the groups. Overall, a specific training methodology has not been identified for approaching a clear demonstration with more effective and low-cost results. However, it seems that a training program based on simplified and short-term CPR combined with instructorstudent feedback, videos, feedback devices, and simulations could cause reduction of the barriers for the lay population to help the OHCA patients and perform CPR.
\end{abstract}

\section{Keywords}

Cardiac arrest; Cardiopulmonary resuscitation; Laypeople; Training

\section{Introduction}

Cardiovascular diseases are recognized as one of the most significant mortality causes around the world. This critical issue has received more attention in Western countries. In such areas, cardiac arrest is the most frequent cause of death [1].

Every year, about 1 in 1000 adults are afflicted by outof-hospital cardiac arrest (OHCA) [2]. The mortality rate of OHCA cases exceeds $90 \%$. Notable, more than half of survivors present some type of neurological sequelae [3].

Cardiorespiratory arrest (CRA) can be potentially reversed by immediate action of basic cardiopulmonary resuscitation (CPR) [3].

Survival of CRA is inversely proportional to the time between CRA and the effective resuscitation action. In fact, 8 to $10 \%$ of the survival chances are significantly reduced by every minute of CPR delay. It is worth to note that resuscitation attempts are usually unsuccessful after 10 minutes of postponement. In addition, high neurological sequelae have been observed after this delay time [4].

Most CRA cases occur in out-of-hospital environments. Obviously, it takes time for emergency services to reach the collapsed victim, in most cases. Therefore, the applied CPR by lay bystanders and first responders plays a key role in saving the patients and obtaining favorable outcome from CRA [5].

It has been reported that only $6 \%$ of the arrest cases could be survived without defibrillators or basic CPRs. While, using semi-automatic external defibrillators (AEDs), as well as CPR training could enhance the survival rate up to $20 \%$. Hence, every person is able to save a life in these situations, without prior knowledge of medicine or healthcare. People of all ages could easily learn compression of chests to simulate the function of the stopped hearts. Moreover, they can use available automated external defibrillators (AEDs), in public places. According to previous studies, basic CPR could be taught to children aged 8 and over. Based upon the obtained data, knowing the role of early action in patient survival has caused voluntary learning of CPR in Spain [1].

Several studies and various scientific societies have recommended training of Basic Cardiopulmonary Resuscitation (B$\mathrm{CPR}$ ) to citizens for reduction of the morbidity and mortality rates. However, their suggestions have not led to a common developed training plan in Europe and even in Spain [6].

The extension of B-CPR knowledge and culture before a possible CRA plays a crucial role in enhancement of the survival rate. So, mass training can be a useful strategy, especially 
in the current situation that even telephone-guided B-CPR with untrained interveners is supported [6].

In European countries such as Denmark, a set of initiatives has been carried out as part of a national campaign such as compulsory BLS training in primary schools for ten years. In this designed plan, training kits were distributed for guiding witnesses through an emergency service telephone-assisted program. Moreover, public access to defibrillation was improved. The results implied double and tripled increment of the survived patients by people assisting in 30 days and 1 year, respectively. The observed results confirmed that the applied initiatives were effective on bystanders of CPR cases. However, this study design did not allow us to determine how much each initiative has contributed to the observed changes [7].

Different initiatives have also been utilized with the aim of massive CPR teaching in Spain. Nevertheless, a few researches have been published. A valuable example is the one carried out in the Municipal Health Center of Chamberí, from 1999 to 2003 . The mentioned study involved 350 students. In another evaluation, the CPR Program was applied to analyze Secondary Education students (PROCES). This program was held in Barcelona in the years 2002 to 2007 and involved 1501 students. Moreover, the training model, conducted in the Almeria province, was performed through massive training sessions in B-CPR. This model focused on the general population, and in a preferential way to specific groups. Public service professionals and students of compulsory secondary education (CSE) revealed the possible B-CPR training of a significant number of citizens through a strategy based on collaboration and concentration of efforts [8].

It is even suggested that more awareness of the need to activate the emergency system may be sufficient, as a simple call may serve to initiate telephone-guided resuscitation techniques [8].

The administration involvement through health and educational services, as well as other public facilities for the citizens are undoubtedly the key building blocks of providing instructions for a significant number of students with a fairly low cost [9].

The involvement of primary care in the development of these courses could contribute to this phenomenon resulting from deep-rooted implantation in the community and its capacity to exercise leadership and influence over it. The B-CPR teaching is already considered as a task that health staffs could offer to the population of their health area through the ESVAP program, in primary care centers [10].

In 2017, a cross-sectional descriptive study was carried out on the attitude and self-perception of the population, in Spain. Based on the results, $99.7 \%$ of the respondents considered it important to train CPR in the population. In addition, evaluation of the effective factors on the CPR onset implied that $64.20,60.30,14.60 \%$ of the respondents, respectively, reflected lack of knowledge, sense of responsibility and fear of harm to the victim, and infection fear. Hence, people illustrated their willingness to do CPR. However, they prefer not to initiate it possibly due to the lack of training and absence of responsibility feeling [1].

CPR training has been effective in enhancement of the re- suscitation maneuver perception, willingness of users to carry it out, and also coordination improvement of the people who perform it.

The main objective of this systematic review is to evaluate the effects of Basic Cardiopulmonary Resuscitation training program and the use of Semi-Automatic External Defibrillation on acquiring and retaining practical skills of lay adults.

\section{Material and method}

\subsection{Design}

A systematic review was designed based upon meta-analysis of the B-CPR training program and the use of AEDs for lay adults to approach greater efficiency in the acquisition and retention of practical skills.

\subsection{Search strategy}

A comprehensive bibliographic evaluation was carried out from December 2019 to March 2020 in the following databases: CINAHL, Cochrane, PubMed, and ScienceDirect. This systematic review followed the recommendations of PRISMA (Preferred Reporting Items for Systematic Reviews and Meta-Analyses).

The raised question, with a PICO structure (Population, Intervention, Comparison, Outcomes), was as follows: do a short training program in $\mathrm{B}-\mathrm{CPR}$, as well as AED management in the lay adult lead to improvement of the knowledge, skills, and attitudes for the Out-of-Hospital Cardiac Arrest management?

To design the search strategy, MeSH descriptors, Decs, and free terms were defined for all keywords. The search strategies have been built with controlled vocabulary terms, text words, synonyms, and related terms for each concept using the most appropriate Boolean operators. The applied search equations are described in Table 1.

Finally, a targeted search strategy was performed to include relevant studies. Then, titles and abstracts of the selected researches were reviewed to assess their conformation to the objectives of the review. Subsequently, the full texts were investigated to determine adjusted studies with the selection criteria.

\subsection{Inclusion and exclusion criteria}

\subsubsection{Participants}

The researches with adult participants of 18 years and over without previous CPR official training were included in this study. Official training is referred to completed procedure of an accredited course or training program.

\subsubsection{Training methodology}

The studies which contained any training program, regardless of the method used for training in CPR and AED use, were considered in the present review.

\subsubsection{Type of study}

Various published Spanish or English articles with analytical or observational experimental research designs were included. The selected articles had a maximum publication age of 10 
TA B L E 1. Description of the utilized search equations.

Cardiopulmonary resuscitation AND training AND laypeople

Cardiopulmonary resuscitation AND training AND population

Cardiac arrest AND Cardiopulmonary resuscitation AND Out-of-hospital cardiac arrest AND Community AND (Education NOT school NOT student)

Out of hospital cardiac arrest AND cardiopulmonary resuscitation AND Community Health Education AND laypeople

Cardiopulmonary resuscitation AND train AND layperson

Cardiopulmonary resuscitation AND train AND laypeople

CPR AND education AND Bystander

years (since 2009). Therefore, the adhered works to the international CPR guidelines, which have been published since 2005, and subsequent ones were included. All rigorous studies linked with the comparison of certain interventions or no intervention in the population were incorporated, regardless of measured or reported outcomes.

\subsubsection{Exclusion criteria}

All studies which were conducted in the school environment or whose participants were less than 18 years old were excluded. In addition, the designed studies based on training of health professionals, as well as health science students (e.g. medicine, dentistry, nursing, or pharmacy) were eliminated. Moreover, researches according with training of professionals with obligatory knowledge in the curriculum of their qualifications related to CPR (lifeguards, firemen, police officers) were removed from this evaluation.

\subsection{Data collection}

Selection and evaluation of the studies were performed by two researchers, named LLL and JMM. It is worth noting that a third investigator (NRF) was consulted and so the consensus was reached in the case of disagreement. The selection of the articles was made in four phases. Identification was considered as the first phase and carried out through searching in different databases, followed by manual exploration, and then removing the repetitive items. Screening was the second step. In this phase, the studies were evaluated by using their titles. Then, appropriate researches were selected based on their titles and abstracts as the third phase. Finally, suitable studies were included and evaluated for estimation of their qualities using the CASP checklist. A coding sheet was used to extract the data from the different selected studies. The final list of included studies was made by consensus by the three reviewers.

Two LLL and JMM coders, independently, extracted the data from the selected studies by using a table, as well as a designed manual coding. The prepared protocol included two instruments. One of them was associated with manual detailing of the guidelines, followed by item coding and scheduling. While, the other one was attributed to preparation of data collection in an excel sheet. The provided data sheet comprised of several information including title, authors, year of publication, research location, design method, recruitment, sample and follow-up, measurement technique, intervention, and bias risk.
During the pilot phase, the applicability of the table and the manual coding was evaluated. So, some modifications were carried out, and then new studies were incorporated. Notably, all researchers agreed with the applied changes.

The data collection sheet contained forty items which were distributed into seven groups, based upon the two coders and third reviewer ideas. Identification, design, participants, intervention, results, quality, and observations were considered as the aforementioned categories.

To facilitate the result management obtained through searching process, a code was assigned to each article. The applied code contained a letter and a number which determined the utilized database for extraction of the study.

An "analysis chart" was created to represent the considered mental map of the present study. In the provided chart, different items have been located according to their contributions to each item.

\subsection{Risk of bias assessment}

The risk of bias was assessed using the Cochrane Collaboration tool. This method is mainly composed of two parts. Seven specific domains are analyzed utilizing this technique, including sequence generation, allocation concealment, blinding, incomplete outcome management, selective reporting, and other biases. Each domain is developed into one or more specific items on a risk table. In the first part, a description is provided linked with the happenings in the study. Whilst, the second part is assigned to identifying the bias risk of each item. The bias risk values are categorized based on "low risk", "high risk", and "moderate or uncertain risk". By applying of this technique tool, risk rate of each study was approached according to the predominated risk obtained in more items. As an example, the study with more low-risk items was considered as low risk. Moreover, the high risk and uncertain risk studies were determined in the same way.

\subsection{Analysis and synthesis}

In order to analyze the quality of researches, the CASP scale was utilized according to the study design and classified in a score range from 1 to 10 . The study with the least quality was scored 1 and so the one with the highest quality was scored 10 . Then, the papers which scored 7 or less were discarded from the investigation. 


\subsubsection{Qualitative synthesis}

A qualitative analysis was conducted to determine the most efficient B-CPR and AED use training program for lay adults in terms of acquisition and retention of practical skills. Notably, the meta-analysis data could be interpreted using the aforementioned analysis. This could be attributed to the heterogeneity of the researches or lack of outcome measurements that made it impossible to include all studies in the meta-analysis.

\subsubsection{Quantitative synthesis}

Evaluation of the analyzed variables of each study was carried out and divided by 4 constructions, including: knowledge, competencies, skills, and performance. The construction performance was also comprised in the meta-analysis due to the lack of homogeneity in the applied instruments in each research. In this construction, the variables of compression/minute ratio, compression depth, and total number of compressions were selected. The studies were designated and included in the meta-analysis when at least two measurement outcomes were obtained from different studies using the same instrument. In order to develop the meta-analysis, the MetaEssentials tool of Excel was utilized [18].

Mean and standard deviation (SD) of both experimental and control groups were calculated in the selected studies to evaluate the sample size effect. The size impact was obtained according to Cohen's proposal. Based upon this method, the difference between average values of the control and experimental groups was firstly calculated. Then, the measured mean difference was divided by the standard deviation of the control group to estimate the size effect. The meta-analysis was based on a random effect model due to the heterogeneity of the studies in terms of their instruments and samples.

The heterogeneity of the selected researches was evaluated using the inferential Q test, proposed by Cochrane, to approach heterogeneity index $\left(\mathrm{I}^{2}\right)$ with $95 \%$ Confidence Interval (CI). Based on this examination, an $\mathrm{I}^{2}$ index greater than $50 \%$ shows high heterogeneity. The inappropriate data for inclusion in the meta-analysis were utilized to assess the association between the different variables. The threshold for the interpretation of the size effect was 0.2 (small), 0.5 (medium), and 0.8 (large). Statistical significance was established with the $P$-value $<0.05$ [19].

\section{Results}

\subsection{Types of studies and the participant characteristics}

In the initial search procedure, 158 articles were identified. During evaluation of these published papers, a series of publications relevant to the objectives of the research have also been identified. These researches provided complementary aspects and were addressed in the considered studies. So, the mentioned documents have then been retrieved through a rhizomatic or secondary search process (Fig. 1).

In the selected studies, the blinding of participants and staff was taken into account to reduce the biased results. This blinding served to alleviate the generated bias by lack of expectations of the control group, regarding the lack of CPR training. In addition, a letter and a number were assigned to each article depending on the search strategies and applied research source. Therefore, retrieval of the included studies was significantly facilitated by the provided well-organized and coded information.

Finally, 10 studies were included which are summarized in Table 1. Two items belonged to quasi-experimental studies and the remaining eight cases were attributed to the randomized controlled clinical trials. The sample size was between 24 and 502 participants. In general, members were lay adults with the average age ranging from 19.5 to 70 years (SD 27.73). Only one of the studies received funding for its completion [15].

All randomized clinical trials followed a sequence of assigning participants to different groups. This corresponds to distinct interventions which in turn involved various CPR and AED training types or different durations of training between the groups. Between the selected randomized clinical trials, examiners/assessors blinding occurred in two trials $[17,21]$. Blinding trials were also selected for the instructors $(n=1)$ [18], participants $(n=1)$ [14], or both groups $(n=1)$ [19].

Participants in most of the selected studies did not receive incentives. However, certification of the course, as an incentive with no cost, was granted to the members in one of the researches. In addition, one option between an American Heart Association (AHA) AED Heartsaver CPR course completion card and 100 USD could be chosen by participants of another study $[17,21]$.

\subsection{Approaches to BLS training and assessment methods}

The included researches followed the most recent resuscitation guidelines of 2010 and 2015. In most studies, training included chest compressions $(\mathrm{CC})$ and ventilation $(\mathrm{V})(\mathrm{n}=3)[12,13,15$, 17, 18]. Although, $\mathrm{CC}$ training without the use of ventilation was applied in two studies [16, 19, 20]. Among the selected investigations, five items comprised the use of AEDs in their training methodologies [12, 13, 15, 18, 21].

Exclusive evaluation of instructor-led training has been reported in a few studies. While, combination and comparison of instructor-led training with other learning or self-learning formulas (e.g. virtual tools with the use of videos or selftraining kits) were included in the most of selected studies.

Training duration in all considered evaluations varied between 60 seconds to 480 minutes. Most studies contained skill practices. In addition, various devices (e.g. training manikins and AEDs) were used to guide CPR during training.

Prior to the intervention, a questionnaire was provided in most studies to collect the socio-demographic data, such as a general rule, age, sex, educational level, and previous training in Basic Life Support (it is an exclusion criterion in the selected studies). The collection of anthropometric data (weight, height, and BMI) stood out in a large part of the evaluated studies.

In most cases, the training evaluation has been carried out through knowledge examinations and especially by simulation with the same devices used in the training program. 


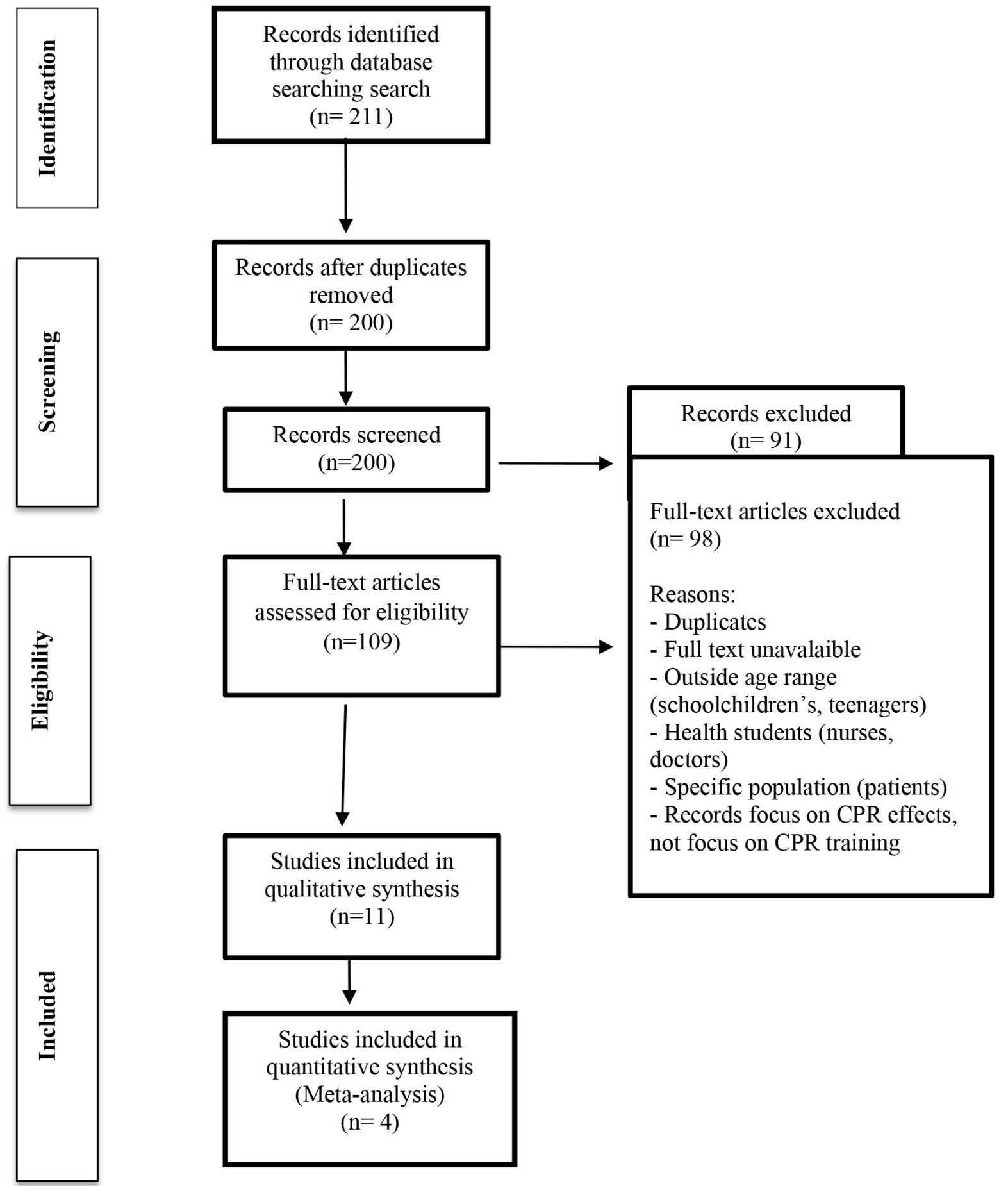

F I G U R E 1. Flow diagram using the PRISMA (Preferred Reporting Items for Systematic Meta-Analyses) guidelines.

\subsection{Thematic categories analyzed in the studies}

\subsubsection{Attitude and self-perception about CPR and AED use}

A cross-sectional descriptive study, performed in our country, claimed that the CPR initiation is mostly influenced by lack of knowledge $(64.20 \% ; \mathrm{n}=233)$, responsibility feeling and fear of harming the victim $(60.30 \% ; \mathrm{n}=219)$, and infection fear $(14.60 \% ; \mathrm{n}=53)$. The knowledge question of "did you know that in the case you do not desire to give mouth-tomouth to a victim, you can help him/her by doing only chest compressions?" was also asked from the participants. The obtained data displayed that $44.5 \%(\mathrm{n}=162)$ of the members knew the vital role of chest compression. Another question revealed that $70 \%(n=250)$ of the participants did not have information about the survival chain. However, concerning the attitude towards OHCA, it should be noted that $80.6 \%$ (n $=291$ ) of the respondents were willing to perform CPR on a stranger. In contrast, in terms of perceived self-efficacy, only $25.9 \%(\mathrm{n}=94)$ felt prepared to do it. In the case of training, $32 \%(\mathrm{n}=116)$ had received CPR training and $85.3 \%(\mathrm{n}=$ $308)$ would like to learn it. Moreover, $99.7 \%(n=361)$ of the respondents considered it important to train the CPR in the 
population.

There was a statistically significant association between the cases who received CPR training, the willing respondents to perform it, the ones with perceived self-efficacy to do it, as well as the members who identified a CPA and initiated CPR $(P<$ 0.001). In all described situations, the percentage was higher in those who experienced training programs [11]. Another trial was conducted in 2017-2018 in the city of Reus (Catalonia), in which $207(50.36 \%)$ of a total participants of 411 responded. According to the obtained results, after training procedure, $89.9 \%$ of the surveyed respondents reported that they knew the OHCA meaning, $86.6 \%$ could identify a victim in CA, and a high percentage of participants were able to recognize public access of AED device.

In the interview on the social impact of CPR training on lay adults, most participants expressed their intention to apply their obtained knowledge and transmit their understandings to others. Moreover, $28.5 \%$ of the participants were at least onetime witnesses of the OHCA. Furthermore, more than half of them (52.5\%) had attempted basic CPR. All participants agreed that the general population should be informed about the location and availability of AED devices in their city and the courses should be taught more frequently [12].

\subsection{Barriers/Limitations for CPR at OHCA performance by laypersons}

In Taiwan, a clinical trial was managed to determine effective factors on the unwillingness of bystanders to perform CPR and improve of the lay public readiness after CPR training. The participants were subjected to a 90 minute training process, consisting of a CPR video teaching (60 min), an AED management $(20 \mathrm{~min})$, and a discussion about CPR legal issues (10 min). Before and after the training, a 22item questionnaire was given to the members. $80 \%$ of the respondents reported their unwillingness to provide mouthto-mouth ventilation because of fearing from legal problems. Prior to CPR training, $36.6 \%$ and $67.8 \%$ of the applicants desired to help a stranger by conventional and hands-only CPR, respectively. After the training, a significant enhancement was observed in the ratio of the participants who were eager to perform conventional CPR. In fact, only $2.19 \%$ of the members reported fearing from legal problems. In addition, fear of further harm was reduced from $76.7 \%$ to $56.0 \%$ after the applied training process. Only 5.84\% reported that they were disinclined to do cardiac compression after attending CPR training. The proportion of unwilling respondents who claimed CPR performing fear was incorrectly doubled from $20.0 \%$ to $40.0 \%$, after training program. Overall, the results of this study showed increment of the CPR performance readiness rate on unfamiliar and familiar individuals after the designed exercise phase. Furthermore, fear of doing more harm was identified as the most significant barrier after the applied training [13].

\subsection{Simplified CPR (CC only) or standard CPR $(\mathrm{CT}+\mathrm{V})$}

A clinical experiment was designed in Singapore in 20132014. In this evaluation, the effectiveness of two standard and simplified CPR training programs was compared on laypeo- ple. The results implied that the simplified CPR group had a significantly higher number of compressions per minute than the standard CPR group (76.45 \pm 26.28 vs. $53.55 \pm 16.91 ; P$ $<0.001)$. Although there was no significant difference in the compression depth of the simplified and standard CPR groups $(36.78 \pm 15.17 \mathrm{~mm}$ vs. $33.24 \pm 11.31 \mathrm{~mm} ; P=0.10)$, the number of compressions with adequate depth was considerably superior in the simplified CPR group than that in the standard CPR group (130.53 \pm 174.23 vs. $33.79 \pm 64.16 ; P<0.01)$.

Overall, proportion comparison of the adequate compressions displayed greater ratio of the simplified CPR group than that of the standard one $(27.74 \pm 34.90 \%$ vs. $9.41 \pm 18.76 \%$; $P<0.01)$. It is important to note that among the participants who performed ventilation, the approached inflation average volume by more than half of the members $(57.14 \%)$ was either too small $(<400 \mathrm{~mL})$ or too high $(>600 \mathrm{~mL})$. Furthermore, the hands-off time was significantly shorter in the simplified CPR group than that in the standard one $(88.35 \pm 52.84 \mathrm{~s}$ vs. $198.52 \pm 60.00 \mathrm{~s} ; P<0.001)[14]$.

\subsection{Instructor-student feedback}

In 2016, an experimental investigation was carried out in Korea. In this examination, two training programs were designed. In the first procedure, conventional CPR $(\mathrm{CC}+\mathrm{V})$ and additional feedback on practical skills were considered for training of the applicants. While, the second program contained CPR teaching solely with hands (no skills practice). The results represented that brief BLS training provided a moderate level of skill for performing CPR and using AEDs. However, high-quality skills for CPR required longer and hands-on training, particularly hands-on training with AEDs.

However, there was a significant difference between the average compression depths resulted from the conventional $\mathrm{CPR}$ training program $(\mathrm{CC}+\mathrm{V})$ combined with AED practice (median: $51 \mathrm{~mm}$, IQR: $44-57 \mathrm{~mm}$ ) and the conventional CPR training program $(\mathrm{CC}+\mathrm{V})$ followed by skills feedback (median: $55 \mathrm{~mm}$, IQR: $50-59 \mathrm{~mm} ; P=0.003$ ). In the case of adequate compression proportion, a significant difference was also observed between the conventional CPR program $(\mathrm{CC}+$ V) with AED practice (median: 30.9\%, IQR: 3.2-69.3\%) and the one accomplished by skill feedback (median: $74.4 \%$, IQR: $24.8-92.9 \% ; P<0.001)[15]$.

\subsection{Use of visual support (algorithm/flowchart) in CPR training}

An Austrian clinical investigation presented the positive effect of algorithm on the obtained results from the trained participants. In a 300 seconds program, the overall time without compression was $147 \pm 30$ and $169 \pm 55$ seconds, respectively, in the groups with and without flowchart $(P=$ 0.024, 95\% CI difference 3.0-41.6).

Concerning the consciousness assessment, the participants trained without and with diagrams performed the process correctly with the ratios of 17 and $88 \%$, respectively. The help request was not made by any participants of the group without algorithm. Whilst, 93\% (39 respondents) of the group members who received the diagram required assistance.

Concerning the action of opening the airway, 9 (22\%) par- 
ticipants of the group without algorithm performed the process. However, it was enhanced to $34(81 \%)$ members in the group that received the flowchart. Breathing assessment was also correctly performed by $15(37 \%)$ applicants and $31(74 \%)$ members of the groups without and with flowchart, respectively.

In the group without flowchart, only $2(5 \%)$ members made the call to the emergency service. However, it was increased to $39(92 \%)$ participants in the group that received diagram.

The total number of chest compressions in 5 minutes was $200 \pm 51$ in the flowchart group, against $189 \pm 110$ respondents in the non-flowchart group $(P=0.55,95 \% \mathrm{CI}-48.7$ to 26.0). Moreover, the depth was slightly greater in the diagram group $(43 \pm 12)$ versus that in the non-diagram one $(41 \pm 12)$.

Furthermore, participants of the flowchart group were much more confident in BLS performance than those who did not receive it $(7 \pm 2$ vs. $5 \pm 2, P=0.0009,95 \%$ CI -2.8 to $0.7)$. Notably, there was not any significant difference between two considered groups in the case of fearing from harming the cardiac arrest victim [16].

\subsection{Self-learning vs. learning in the classroom}

Self-learning is proposed as a useful method to bring knowledge to a wide number of individuals at an affordable cost. Several studies reported no significant difference between a self-learning method and a classroom one, regarding to the OHCA learning management.

A clinical trial was carried out on the local Hong Kong lay population. In this examination, the effects of selfinstructional CPR video and traditional classroom instruction were evaluated on the applicants. The self-study members received a kit with an inflatable manikin, a 5-minute DVD, and an instruction manual. While, the classroom group participants received an instruction manual and access to the face-to-face sessions with an instructor. The second group was asked to take a practice test immediately after the classroom training. Participants in the self-study group were also required to return for a practical test at a mutually agreed time to pass the same test. One year after the intervention, both group members who were successful in the test were invited to take a new exam.

After the training step, both groups showed a significant increment in the number of willing members for applying CPR on acquaintances or strangers.

In the self-study group, $112(90 \%)$ participants were successful in the exam. Whilst, 125 (95\%) applicants of the classroom learning group passed the test. Although the classroom learning group turned out to be better in the skill performance, no statistically significant difference was observed between the pass rates of two considered groups.

In addition, at least $50 \%$ of the applicants were satisfactory familiar with CPR skills after 1 year. Notably, there was no statistical difference between the two groups in this case.

In relation to the self-learning group, more than $98 \%$ of the manikins were still working after 6 months. Notably, 28 (23\%) respondents had taught CPR to their friends or family members in the last 6 months. Generally, opinions about the Self-
Training Kit were very favorable; about $95 \%$ of the participants considered it as a very good or good training technique. Also, over $85 \%$ of respondents desired to try self-study process again and also recommend it to others.

The results implied that the CPR self-learning efficiency among laypeople is equivalent to efficiency of attending a faceto-face training program. The self-learning program provides the virtue of allowing the student more time for study and practice, unlimited repetition, and the feasibility of teaching acquaintances [17].

\subsection{Learning with feedback devices}

In 2013-2014, an examination was performed in Italy to evaluate the feedback of applied devices on the chest compression quality during CPR training of lay adults. In the mentioned study, 450 participants were involved. In a random selection, one group received training without feedback, while two groups experienced feedback with training manikins that lasted 1 and 10 minutes, respectively. The results exhibited significant improvement of chest compression depth $(P$ $=0.012)$, chest retraction $(P<0.001)$, and total CPR score $(P<0.001)$ in the feedback groups compared to those of the group members who did not use any feedback. Moreover, the frequency of CCs was similar $(P=0.529)$ in all three groups. Furthermore, there was not any significant difference between two groups with brief and prolonged feedbacks. So, it can be inferred that only 1 minute of real-time visual feedback could be sufficient for laypeople to achieve better CPR quality [18].

\subsection{Using videos for learning of lay adult CPR}

A $1: 1: 1$ randomized experimental investigation was performed in Arizona to demonstrate the ability of learning, demonstration, and retaining effective hands-on CPR skills through watching a 60 -second ultra-short training by adults without prior formal CPR training. The trial compared the results of a control group, which received no training intervention, with various trained groups based on watching a video. The video contained assisting an adult with a sudden collapse using hands-only CPR. The subtitled video guided the first group of participants to call 911 and press hard and fast. The second group of respondents was trained through a 5-minute video, in which the training procedure was shown by an instructor. The last group watched the same video, followed by 3 minutes of psychomotor skill practice on an inflatable manikin.

Based on the obtained data, $23.5 \%$ of the members in the control group did not attempt to perform CPR in the designed scenario. While, all members in the trained groups tried to assist. The average compression rate in the "ultra-short video" group (60 seconds long) was significantly higher than that of the control group $(P=0.007)$. Moreover, significantly improved rates were observed in the "short video" group (5 min duration) and the "short video with practice" group (5 min video and 3 min practice) in comparison with that of the control group ( $P=0.015$ and $P=0.010$, respectively).

The average compression depth was estimated at $39 \mathrm{~mm}$ in the control group after excluding those who did not perform 
TA B L E 2. Main characteristics of the clinical trials included in the review: study population and training methodology.

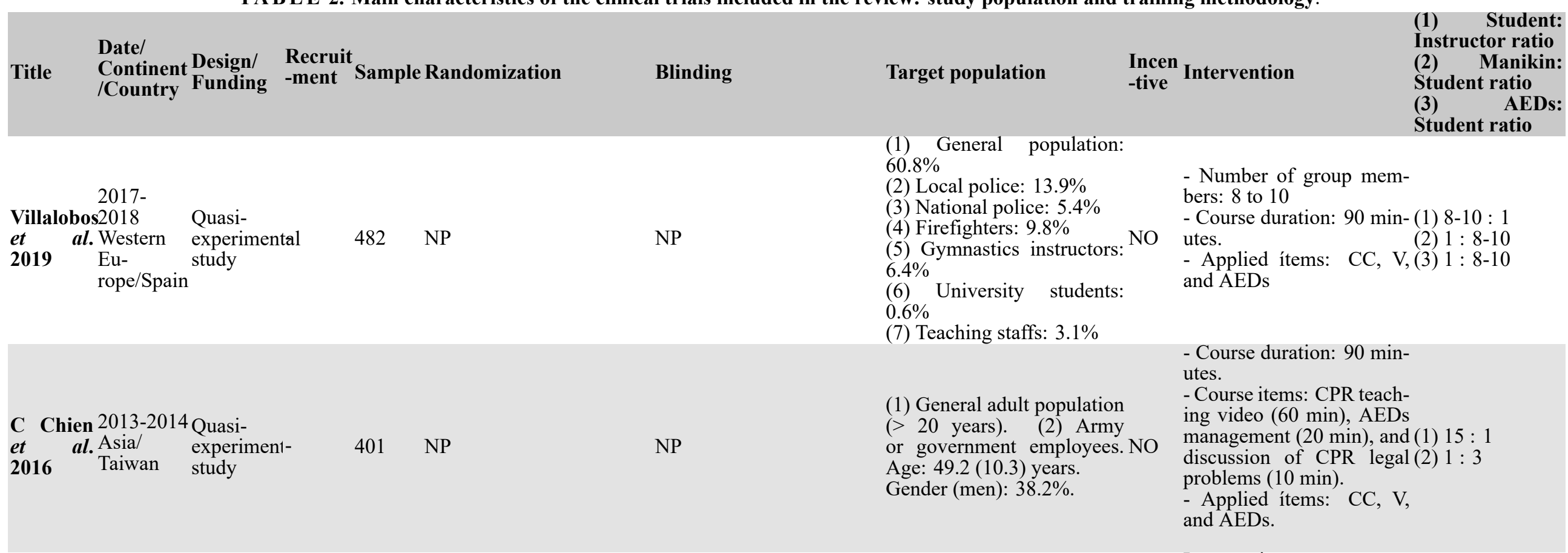

Subject assignments to 1 of

the following groups:

- Group C (control): $\mathrm{n}=51$

- Group USV (ultra short

Subjects and instructors training in the previous 2

- SV Group (short video): $n$ were not aware of the years, hypothesis or design of the (3) English fluent.

$\begin{array}{lll}\text { Bobrow North } & \text { Randomized } \\ \text { et } & \text { al. America/ } & \text { controlled } \\ \mathbf{2 0 1 1} & \text { USA } & \text { clinical }\end{array}$

336

/Arizona tria
$=99$

- SVP Group (short video stur

Age: Control = 44.8 (11.8);

with practice): $\mathrm{n}=91 \quad$ The performance recorders Immediate $=48.0(14.8) ;$

All groups except group $\mathrm{C}$ were blinded to the type of Delayed $=46.9(13.4)$

participants were random- training. Sex (male): Control $=23$

lytested immediately $(\mathrm{n}=$

45.1);

lytested immediately $(\mathrm{n}=$
that day or returned 2

(44.4); Delayed = 69 (48.3)

months later for examina-
Intervention :

- According to the randomization group : subjects did not receive training intervention in group $C$,

subjects watched an ultrashort 60 -second video produced without skill practice in the USV group, Not specified respondents watched a 5minute video without skill practice in the SV group, and subjects experienced an 8-minute video with an 8-minute video with
$\mathrm{CPR}$ practice during visualization in the SVP group. - Applied ítem: CC 
TA B L E 2. Continued.

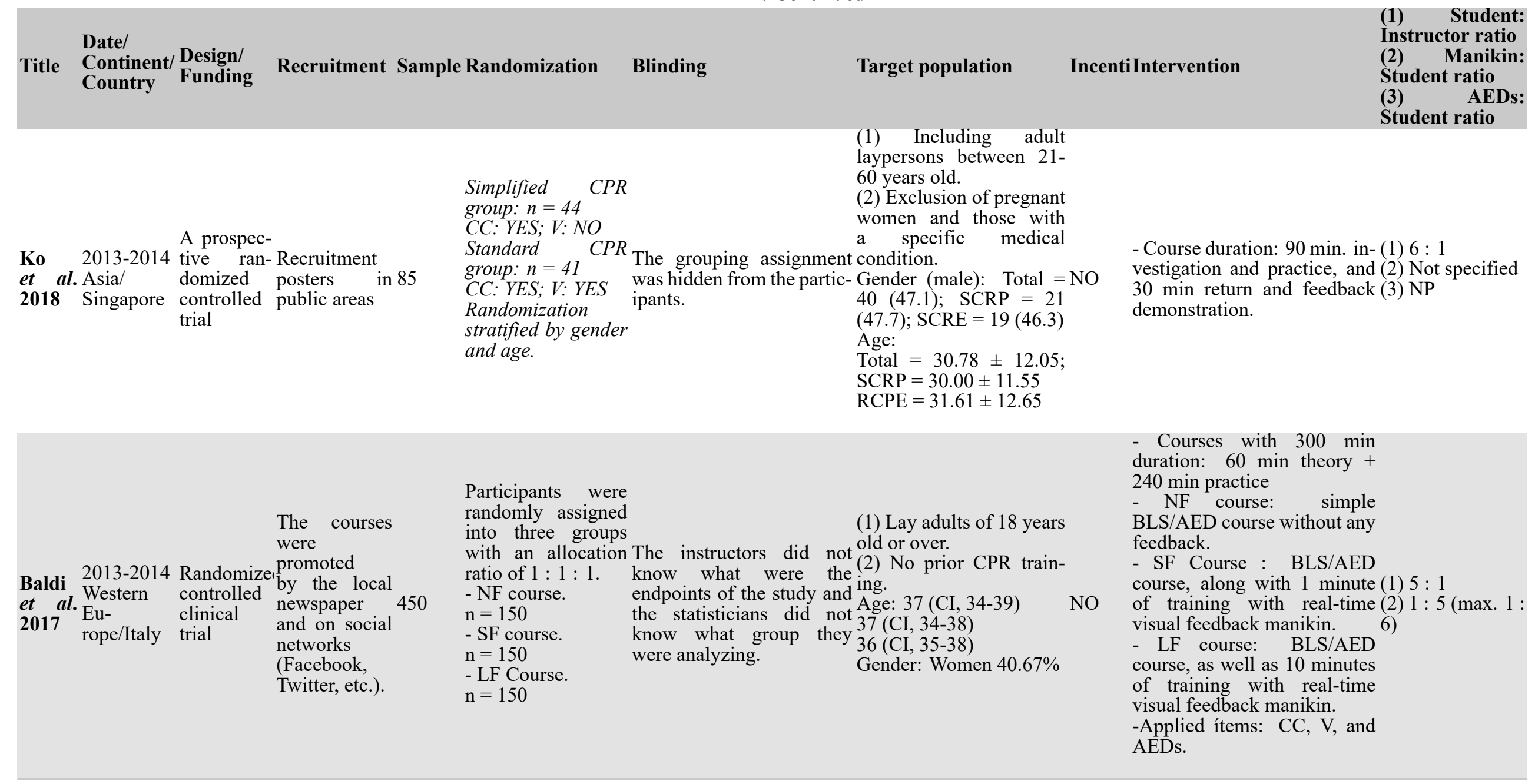




\section{Recruit}

-ment

Sample Randomization Blinding

\section{Target population}

(1) Student: In-

(1) Student:

structor ratio

dent ratio

(3) AEDs: Stu-

dent ratio

- Self-learning group: Participants received a kit with an inflatable manikin, a 5-minute

Randomly The examiners (1) Lay adults between the ages of 18 blinded to 70 years old. Male to female ratio YES, DVD, and an instruction manChung 2007-2008 et al. Asia/ prospective randomized, 2010 Hong Kong $\begin{aligned} & \text { single-blind } \\ & \text { controlled }\end{aligned}$ 2 groups: - Self- to the received was 132: 124 Age range: 18 to 62 free ual.

learning group $n=$ training by the (average $=39$ and standard deviation course - Classroom Group: Participant 124 - Classroom participants = 10 years). received an instruction manual
Women of 45 years old or over, mainly housewives, with no regular income.

Tae 2014-2015 Randomized, takes place into 2 groups :

2014-2015 Randomized, at a local - Group S-BLS

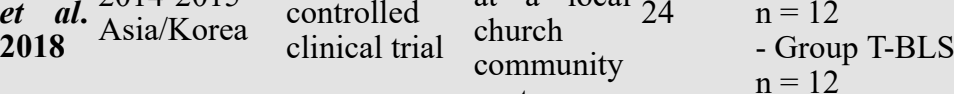

Age : S-BLS : 59 (5\% DS); TBLS : 53 (6\% DS)

Height: S-BLS: $159.7 \mathrm{~cm}(4.2 \% \mathrm{DS})$

T-BLS: $158.3 \mathrm{~cm}(3.7 \% \mathrm{DS})$

Weight: S-BLS: $57.1 \mathrm{~kg}(9.9 \% \mathrm{DS})$ T-BLS: $54.9 \mathrm{~kg}(6.6 \% \mathrm{DS})$ and assistance to the classroom. - Applied ítems: CC and V.

- Group S-BLS: 30 min videobased self-instruction with a dummy for each participant, as well as 30 min information and practice session with the course instructors.

no simulation.

(1) Not specified Group TBLS: 30 min video (2) $24: 24$ (both based self-instruction plus 15 groups)

min simulation and 15 min brief-

ing with the course instructors.

Simulations

- Applied ítem : CC

Volunteers between 18 and 80 years of age, regardless of gender, from a non-medical profession.

Rössler2011-2012 prospective

et al. Europe/ randomized, -

2013 Austria divided into 2

groups:

Group A : With

flowchart support. NO

$\mathrm{n}=41$

Group B: No

flowchart support.

$\mathrm{n}=42$
Age: CPR without support: 34 \pm 12 ; and CPR with support: $39 \pm 11$ Gender (female): CPR without NO support: $13(32 \%)$; and CPR with support: $13(30 \%)$

Years since last course: CPR without support: $12 \pm 10$; and CPR with support: $15 \pm 12$
- Group A: Perform CPR with flowchart support. These participants received the chart right at the beginning of the scenario without further instructions on its contents.

- Group B: Perform CPR with- Not specified out flowchart support.

- Both groups did not receive any additional presentations or support.

- Applied ítem: CC 
TA B L E 2. Continued.

Both groups received a sixstation BLS course.

YES ML Group: After AHA video

- Randomly placed in to 2 groups:

Clinical,

2013-2014 controlled, Recruitment Boet North Americaprospective, in the et al. Canada/ quasi- Faculty of 49 2017 Ottawa randomized Science. trial
Control group (time-based

BLS course $(\mathrm{TB})$

$\mathrm{n}=22$

Group intervention (mas- to

Group intervention (mas- to

(ML):

$\mathrm{n}=27$
(1) Undergraduate students Partici without formal CPR training, 2 options:

i.e., lay adults.

instruction, each participant deThe evaluators Gender (female): TB Group American Heart level of mastery was achieved

$\begin{array}{ll}\text { Association for each of the six stations. } & \text { (1) } 5: 1 \\ \text { AHA) AED TB Group: } 10 \text { minutes of intro- } & \text { (2) } 1: 1\end{array}$ duction followed by 90 minutes $\begin{array}{ll}\text { Age (years): } & \text { Heartsaver duction followed by } 90 \text { minutes } \\ \text { TB Group } 19.5 \text { [ } 18.0 \text { - 21.0]; CPA course of actual course content, with }\end{array}$ and completion card additional time for feedback as ML Group 20.0 [18.75 - 21.0]. (BLS-A) or needed up to a maximum of 2 (2) $\$ 100 \quad$ hours total.

- Applied ítems: CC, V, and AEDs.

\begin{tabular}{|c|c|c|c|c|c|}
\hline $\begin{array}{ll}\text { Jin } & 2015 \\
\text { et } a l . & \text { Asia/Korea } \\
\mathbf{2 0 1 6} & \text { Asia }\end{array}$ & $\begin{array}{l}\text { A } \\
\text { prospective } \\
\text { randomized } \\
\text { controlled } \\
\text { trial. Funded } \\
\text { by the Faculty } \\
\text { Korean } \\
\text { Centers for } \\
\text { Disease } \\
\text { Control and } \\
\text { Prevention }\end{array}$ & 502 & $\begin{array}{l}\text { - Randomly divided by } 4 \\
\text { training levels: } \\
\text { Level } 1: \text { CPR with hands } \\
\text { only ( } 40 \text { min). } \\
\mathrm{n}=121 \\
\text { Level } 2: \text { CPR with hands } \\
\text { only and practice with } \\
\text { AED ( } 80 \text { min). } \\
\mathrm{n}=119 \\
\text { Level } 3: \text { Conventional NO } \\
\text { CPR (CC and V) and prac- } \\
\text { tices with AED (120 min). } \\
\mathrm{n}=124 \\
\text { Level 4: Conventional } \\
\text { CPR, same as Level } 3 \text { and } \\
\text { comments on CPR and } \\
\text { AED skills ( } 180 \text { min). } \\
n=121\end{array}$ & 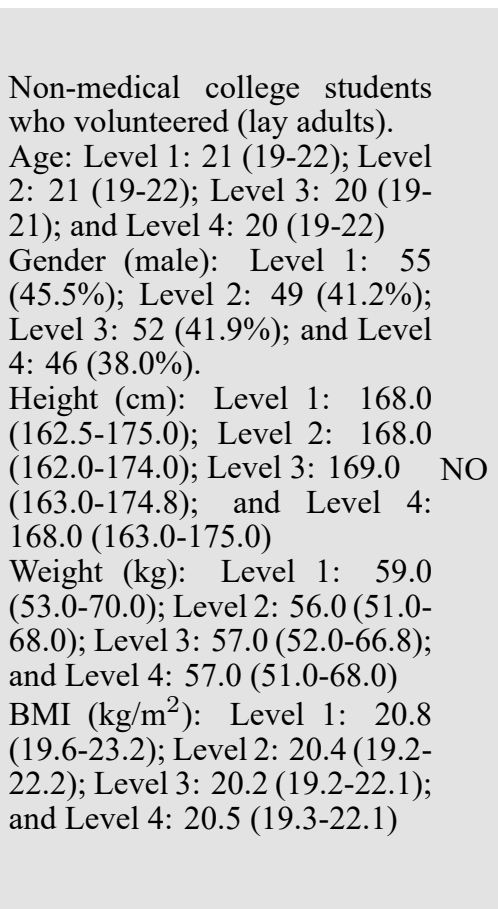 & $\begin{array}{l}16 \text { courses (4 courses for each } \\
\text { level) } \\
\text { Level 1: Recognizing cardiac } \\
\text { arrest and calling for help, } \\
\text { chest compressions, practice } \\
\text { for hands-only CPR, presenting } \\
\text { an AED, and how to use it, } \\
\text { presenting mouth-to-mouth } \\
\text { resuscitation, and a summary of } \\
\text { the course. } \\
\text { Level } 2 \text { (Hands-only CPR, } 80 \\
\text { min): all Level } 1 \text { contents, as } \\
\text { well as practice with an AED. } 6: 1 \\
\text { Level } 3 \text { (Conventional CPR, (2) } 1: 2 \\
120 \text { min): All contents of Level } \\
2 \text { ( } 1: 2 \\
2 \text {, and the practice of mouth- } \\
\text { to-mouth breathing, along with } \\
\text { conventional practice with an } \\
\text { AED. } \\
\text { Level } 4 \text { (Conventional CPR, } \\
180 \text { min): All Level } 3 \text { content, } \\
\text { as well as BLS skill test using an } \\
\text { AED, and providing feedback } \\
\text { on CPR and AED skills. } \\
\text { - Applied items: CC, V, and } \\
\text { AEDs. }\end{array}$ \\
\hline
\end{tabular}


chest compressions. The "short video with practice" group had a significantly greater median depth of compression than the control group $(P=0.0003)$.

The respondents of the "short video" and "short video with practice" groups were more likely to call 911 than those of the control one.

The observed results suggest that the 60 seconds of CPR video encourage people to attempt hands-only CPR. In addition, applying this technique causes superior skills in the participants compared to those in the untrained people. The aforementioned method is short enough to be used in a large number of media outlets, and then it could provide public education for enhancement of the CPR rate and so OHCA survival [19].

\subsection{Intervention duration (training program)}

A quasi-experimental study was carried out with the aim of training lay adults to provide skills in basic CPR and the use of an AED, in our country and specifically in Reus (Catalonia). Thirty-six 90-minute training courses were held, in which 482 participants were distributed in groups of 8 to 10 members.

At the beginning and the end of the training courses, knowledge level of the members regarding to CPR and AED basic uses was evaluated by using an adapted questionnaire. At the end of the courses, a practical evaluation of the BLS sequence was also carried out. In this examination, several elements were measured, including: (a) check the scene, (b) assess the consciousness level, (c) open airway, (d) monitor spontaneous breathing, (e) call for help, (f) chest compressions (quality), (g) compression frequency, (h) two ventilations (quality), and (i) alternate compressions/vaccinations. The value of each item was considered one point. Students performing at least 8 items were known successful in the considered evaluation.

According to the obtained data, 419 members displayed adequate scores and 63 had inadequate ones. By the end of the courses, knowledge level was significantly improved (from $3.17 \pm 1.2$ to $3.80 \pm 1.1$ points, $P=0.001)$ and an increment was observed in the participant percentage who answered all questions of the questionnaire.

$86.9 \%$ of the trained participants acquired adequate CPR skills. In addition, the results showed that a significant percentage of the applicants received sufficient skills in the CPR and AED basic use, which confirms the usefulness and effectiveness of the designed courses.

Most participants were satisfied with the training courses, expressed their intention to apply their acquired knowledge, and considered it necessary to teach their understandings to others [12].

\subsection{Use of simulations in CPR training programs for laypeople}

A clinical investigation, conducted in Korea in the years 20142015, considered that in occurred cardiac arrests at homes, the witnesses are usually older or middle-aged housewives $(\mathrm{n}=$ 19). Based on this, two groups of participants were randomly created. Both groups underwent 1-hour training and were trained by a 30 -minute video. In addition to the video, one group of participants received a 30-minute CPR skill practice (only CC), while the other one experienced simulations or roleplaying.

During the initial simulation, the average of the total time without flow (IQR) was obtained 79.6 (66.4-96.9) and 147.6 (122.5-184.0) seconds for the training group with and without simulations, respectively $(P<0.01)$. More chest compressions were performed by the group who experienced simulations or role-playing.

Mean chest compression ratio with adequate depth appeared to be lower in the group with simulation than that in the nonsimulation training group. However, the difference was not statistically significant $(12.1$ vs. $23.7, P=0.41$, in the initial simulation and 35.0 vs. $24.4, P=0.87$, at 6 months of followup). There was also no significant difference between both groups in the case of chest compression average rate.

Overall, the results illustrated that the trained participants with the simulation training program had a shorter no-flow time and fewer interruptions during the CPR simulation compared to the members of the non-simulation group [20].

\subsection{Quantitative analysis}

Three meta-analyses were performed among the ten selected studies. Fig. 3 to 5, respectively, illustrate quantitatively analyzed variables of compression/min ratio, compression depth, and a total number of compressions.

The results showed low heterogeneity $\left(\mathrm{I}^{2}=0 \%\right)$, as well as a non-significant result for the combined effect of the included studies in the case of compression $/ \mathrm{min}$ ratio $(\mathrm{n}=4 ; \mathrm{z}=0.14$; $P=0.891$ ) (Fig. 2).

For the depth variable, the results represented low heterogeneity $\left(\mathrm{I}^{2}=0 \%\right)$. In addition, the combined effect of the studies, included for this variable $(\mathrm{n}=3)$, exhibited no significant result for the MA $(\mathrm{z}=1.20 ; P=0.232)$ (Fig. 3$)$.

The results also showed low heterogeneity $\left(\mathrm{I}^{2}=0 \%\right)$ for the total number of compressions. This implied a non-significant result $(\mathrm{z}=0.26 ; P=0.794)$ for the included studies regarding to evaluation of this variable $(n=4)($ Fig. 4$)$.

\subsection{Assessment of risk of bias}

The bias parameter was evaluated following the guidelines adjusted by the Cochrane collaboration tool. According to the instruction, seven items were evaluated (see Table 2).

The "Sequence generation" item was categorized into three levels containing $60 \%(\mathrm{n}=6)$ low risk, $10 \%(\mathrm{n}=1)$ moderate/ unclear risk, and $30 \%(\mathrm{n}=3)$ high risk.

In the case of "allocation concealment" element, none of the selected studies were considered as low risk. While, $70 \%(\mathrm{n}=$ 7) were assumed as moderate/uncertain risk and 30\% $(n=3)$ were identified as high risk.

$10 \%(n=1), 60 \%(n=6)$, and $30 \%(n=3)$ of the investigated studies were categorized into three risk levels of low, moderate, and high risks in the case of "Blinding of participants and staff" issue, respectively. In the matter of "blinding of the evaluators", $10 \%(n=1)$ and $90 \%(n=9)$ of the evaluations were identified, respectively, as low and moderate/uncertain risk levels. Moreover, $80 \%(n=8)$ of the researches did not provide sufficient information on possible biases and $20 \%$ (n 


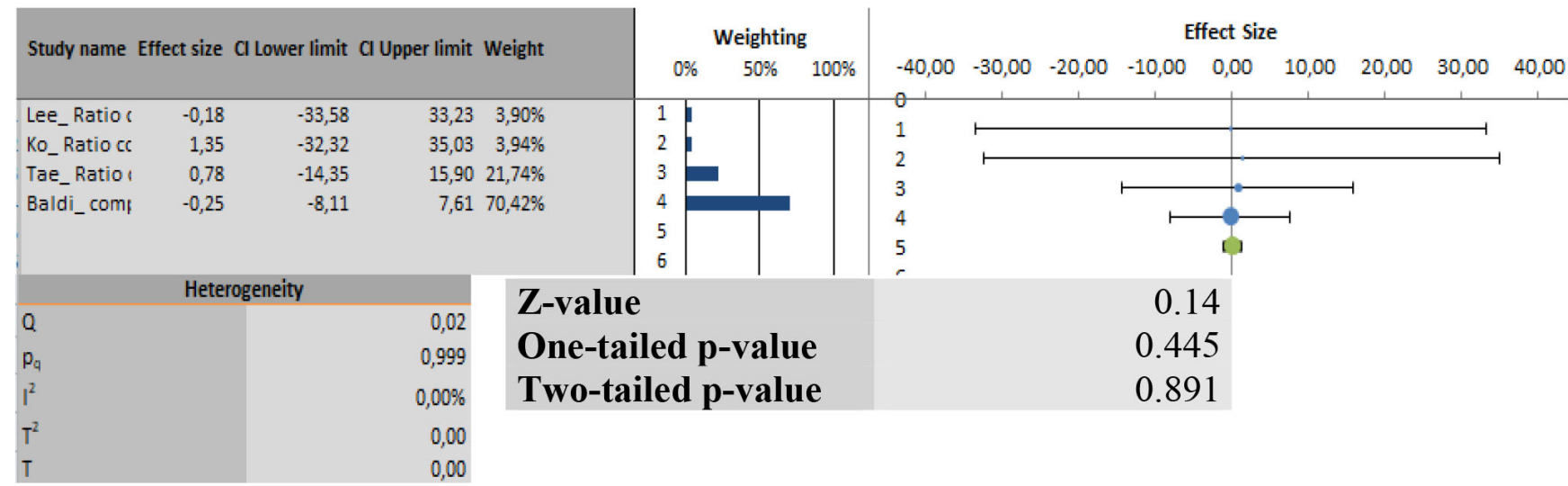

F I G U R E 2. Forest plot for comparison of compressions/min ratio variable in different cardiopulmonary resuscitation teaching methodologies.

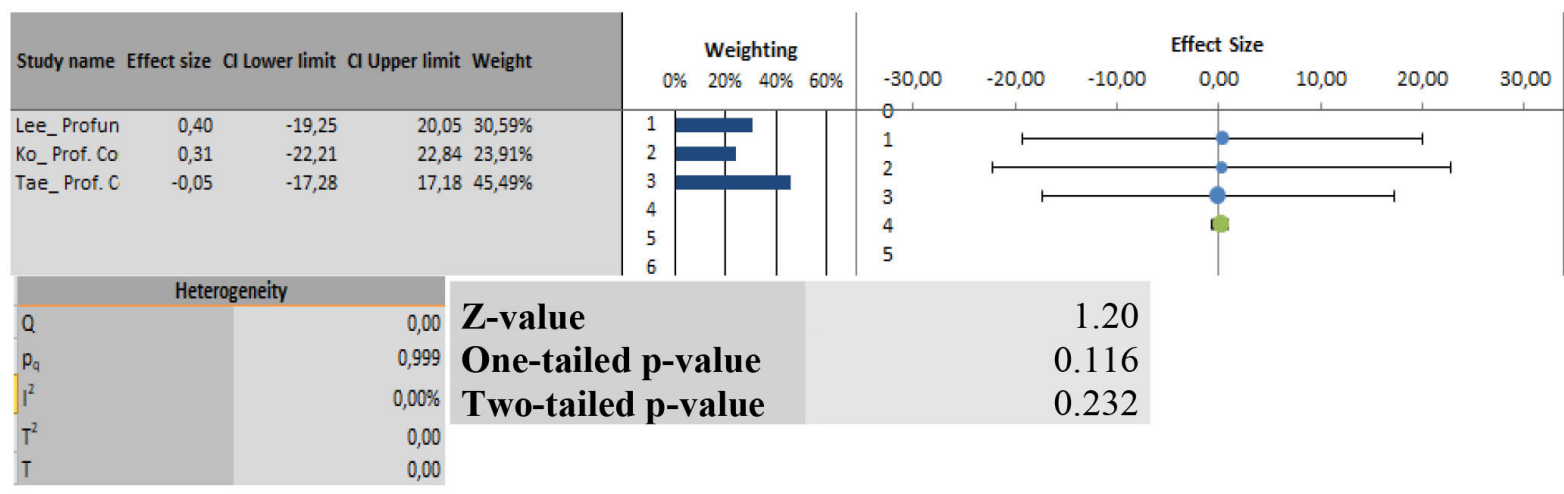

F I G URE 3. Forest plot showing comparison of depth of compressions depth variable in various cardiopulmonary teaching methodologies.

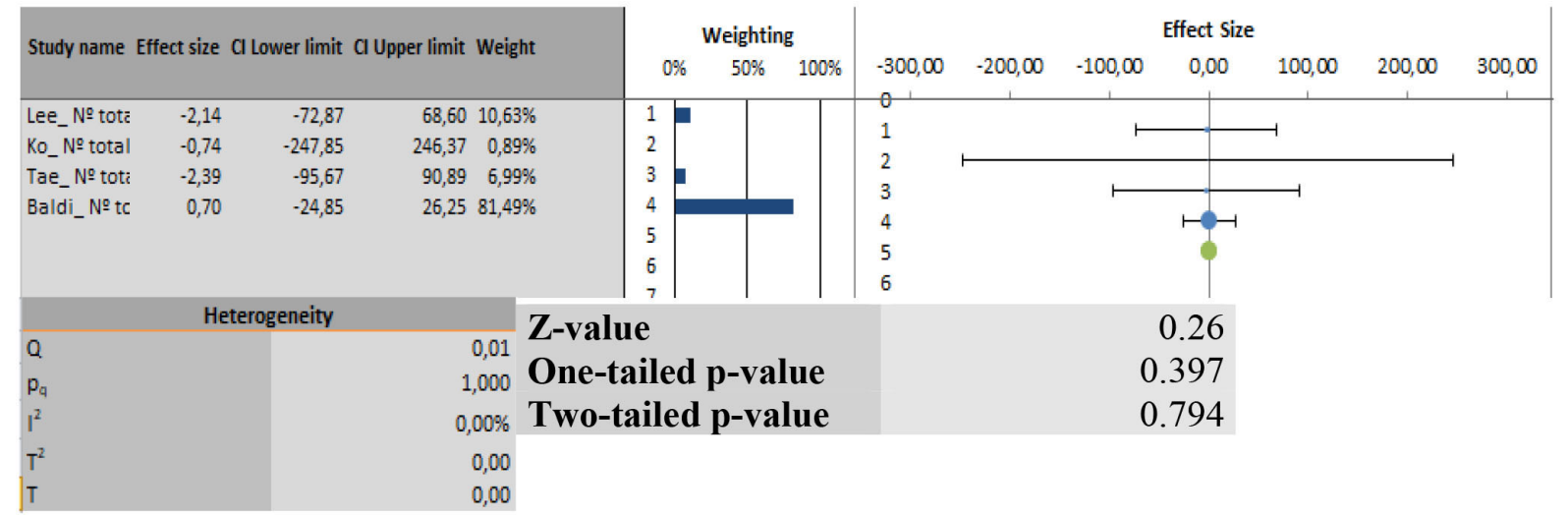

F I G U R E 4. Forest plot displaying comparison of total number of compressions in different cardiopulmonary teaching methodologies.

$=2)$ of them had a moderate risk.

For the issue of "selective reporting of results", none of the selected studies were considered as moderate risk. However, $80 \%(\mathrm{n}=8)$ and $20 \%(\mathrm{n}=2)$ were determined as low and high risk levels, respectively.

\section{Discussion}

In the present symmetric review, the heterogeneity of the included studies hampered the establishment of definitive recommendations on the optimal strategy for training lay adults in BLS. Variability in the contents and methodology of the interventions, duration, tools, utilized devices, variables, and evaluation time made it difficult to approach a reliable comparison between the researches. In addition, different evalua- 


\section{Risk of bias summary}

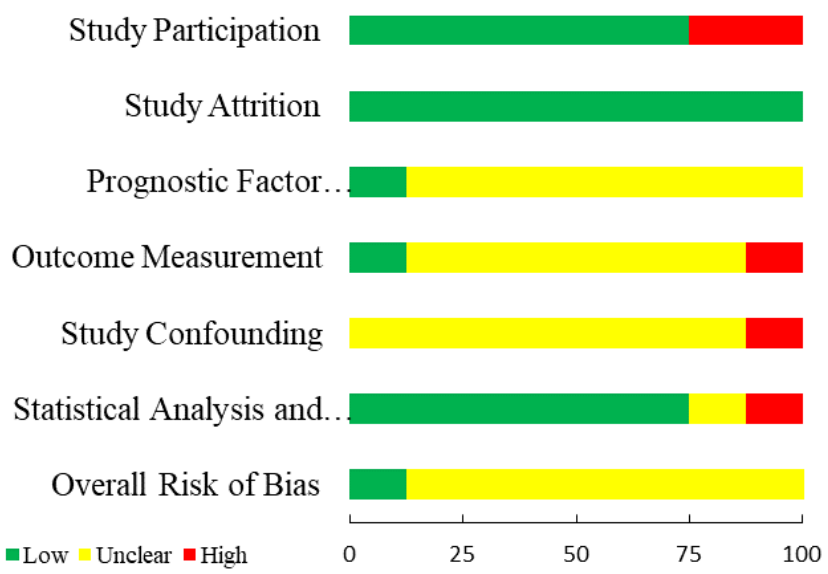

F I G U R E 5. Assessment of bias risk.

tion protocols and quality indicators were applied. However, there was a significant association between the received CPR training and perceived self-efficacy in CRA identification and CPR initiating.

Similarly, there are few published studies containing the AED use exercise in their designed plan [12, 13, 15, 18, 21]. Even, more scarce cases documented these skills after the training procedure. There is evidence supporting the association of early defibrillation by witnesses and better clinical results. However, there is no common regulation for the use of AEDs by citizens in all Autonomous Communities of our country. In most cases, no data was provided regarding the validity of the assessment instruments.

The disinclination of the members to provide mouth-tomouth ventilation, fear of legal problems and further harm fright has been the main barriers that limited the CPR performance by the lay population. Nevertheless, these blocks have declined after training programs. Based on the literature, the proportion of willing participants to perform conventional CPR has been significantly increased by applying a training step. However, fear of causing more harm has remained the most important barrier even after the training procedure [13].

Most studies analyzed the quality of CPR by focusing only on CCs [14, 19, 20].

It seems more effective to opt training process based upon the simplified CPR (CC only). Since, this method has demonstrated significantly more compressions per minute, as well as a greater number of compressions with adequate depth than the standard CPR group $(\mathrm{CC}+\mathrm{V})$ [14]. In addition, most of the participants who performed insufflation or respiratory ventilation maneuvers presented inadequate results, such as low or very high volume of insufflation. These results are consistent with the current recommendations that support the simplified approach of promoting compression-only CPR training for laypeople [14].

The necessity of accessible learning for a great number of citizens has led to the creation of new formulas, such as selftraining as an alternative for traditional courses. Overall, the self-learning program provides the opportunity for more study and practice, unlimited repetition, and feasible teaching of acquaintances [17].

Algorithm usage before the training process also seems to have beneficial results. Based on the revealed data, the time without compressions is reduced in those participants who received this support. Providing a flowchart to participants improves several vital issues including the correct performance of the consciousness assessment maneuvers, EMS alert, airway opening maneuver, and breathe assessment. The total number of compressions and their depths are also better through distribution of flowcharts prior to the face-to-face training [16]. The use of feedback devices during CPR training in lay adults causes improvement of the chest compression quality. Moreover, several researches which applied very short duration feedbacks are in well-agreement with this conclusion [18].

Adults without prior formal CPR training can learn, demonstrate, and keep effective hands-only CPR skills through watching a 60 -second ultra-short training video. So, this method of education holds promise for increment of CPR rates and survival from OHCA. Concerning training formulas with different durations, the short formulas can reasonably improve the skills compared to the longer duration methodologies. Nevertheless, it is impossible to make precise conclusions about determining the efficient and feasible duration of a training program in CPR and AED use. This could be attributed to the diversity of contents and evaluation methods [19].

Based on a case study, a simulation training program could lead to CPR simulation with a shorter time, without blood circulation, and fewer interruptions [20].

\section{Conclusions}

In conclusion, CPR training increases the perceived CPR selfefficacy and willingness to perform it. In addition, it enhances the number of people who desire to perform it.

A training methodology leading to a clear demonstration with more effective and affordable results has not identified yet. However, it seems that combination of a training program based on simplified and short-term CPR with instructorstudent feedback, videos, feedback devices, and simulations could help to reduce the barriers for the lay population to assist the OHCA patients and perform CPR.

Self-learning methods have demonstrated comparable results than face-to-face learning techniques. The time reduction, along with the cost-effective procedure allows simultaneous training to a larger number of people.

The implementation of skills with the use of manikins, instructor-student feedback, and face-to-face simulations enhances the quality of the obtained results. So, methodology combinations in this training field could be a valuable approach.

\section{AUTHOR CONTRIBUTIONS}

Conceptualization: Lourdes Luque (L.L.-L.) and Jesús Molina (J.M.-M.); methodology, L.L.-L. and J.M.-M..; validation, J.M.-M. ; formal analysis, L.L.-L. and J.M.-M.; investigation, L.L.-L.; data curation, J.M.-M..; writing-original draft 
preparation, L.L.-L.; writing-review and editing, J.M.-M. and L.L.-L; supervision, J.M.-M.; project administration, J.M.M.; All authors have read and agreed to the published version of the manuscript.

\section{ACKNOWLEDGMENT}

I would like to express my gratitude to all those who helped me during the writing of this manuscript. Thanks to all the peer reviewers for their opinions and suggestions.

\section{CONFLICT OF INTEREST}

The authors declare that there is no conflict of interest regarding the publication of this article.

\section{REFERENCES}

[1] Santiago I, Beaumont C. Eduquemos a toda la población en reanimación cardiopulmonar. Anales del Sistema Sanitario de Navarra. 2018; 41: 285286. (In Spanish)

[2] Baldi E, Contri E, Burkart R, Borrelli P, Ferraro OE, Tonani M, et al. Protocol of a multicenter international randomized controlled manikin study on different protocols of cardiopulmonary resuscitation for laypeople (MANI-CPR). BMJ Open. 2018; 8: e019723.

[3] Miró Ò, Díaz N, Escalada X, Pérez Pueyo FJ, Sánchez M. Review of initiatives carried out in Spain to implement teaching of basic cardiopulmonary reanimation in schools. Anales del Sistema Sanitario de Navarra. 2012; 35: 477-486. (In Spanish)

[4] Caparrós L, Dolores M. Nivel de conocimiento y aptitudes de la reanimación cardiopulmonar en trabajadores. Revista Enfermería del Trabajo. 2017; 7: 109-116. (In Spanish)

[5] Böttiger BW, Lockey A, Aickin R, Castren M, de Caen A, Escalante R, et al. "all citizens of the world can save a life"-the World Restart a Heart (WRAH) initiative starts in 2018. Resuscitation. 2018; 128: 188-190.

[6] García del Águila F, López Rebollo E, Rosell Ortiz F, Mellado Vergel F, Alba Resina Á, Plaza Picón G. Strategia para la formación masiva en reanimación cardiopulmonar básica a primeros intervinientes. Prehospital Emergency Care. 2011; 4: 205-208. (In Spanish)

[7] Wissenberg M, Lippert FK, Folke F, Weeke P, Hansen CM, Christensen $\mathrm{EF}$, et al. Association of national initiatives to improve cardiac arrest management with rates of bystander intervention and patient survival after out-of-hospital cardiac arrest. Journal of the American Medical Association. 2013; 310: 1377-1384.

[8] López-Messa JB, Martín-Hernández H, Pérez-Vela JL, Molina-Latorre R, Herrero-Ansola P. Novelities in resuscitation training methods. Medicina Intensiva. 2011; 35: 433-441.

[9] García del Águila F, López Rebollo E, Rosell Ortiz F, Mellado Vergel F, Alba Resina Á, Plaza Picón G. Strategia para la formación masiva en reanimación cardiopulmonar básica a primeros intervinientes. Prehospital Emergency Care. 2011; 4: 205-208. (In Spanish)
[10] Miró Ò. Elementos para mejorar la difusión de la reanimación cardiopulmonar entre la población lega. AtencióN Primaria. 2013; 45: 544-545. (In Spanish)

[11] Martínez C, Carmen M. Actitud y autopercepción de la población en la cadena de supervivencia. Metas de Enfermería. 2017; 20: 49-54. (In Spanish)

[12] Villalobos F, Del Pozo A, Rey-Reñones C, Granado-Font E, SabatéLissner D, Poblet-Calaf C, et al. Lay people training in CPR and in the use of an automated external defibrillator, and its social impact: a community health study. International Journal of Environmental Research and Public Health. 2019; 16: 2870.

[13] Chien CY, Weng YM, Hsu SC, Kuo SW, Chaou CH. Effect of population-based training programs on bystander willingness to perform cardiopulmonary resuscitation. Signa Vitae. 2016; 12: 63-69.

[14] Ko RJM, Lim SH, Wu VX, Leong TY, Liaw SY. Easy-to-learn cardiopulmonary resuscitation training programme: a randomised controlled trial on laypeople's resuscitation performance. Singapore Medical Journal. 2018; 59: 217-223.

[15] Lee JH, Cho Y, Kang KH, Cho GC, Song KJ, Lee CH. The effect of the duration of basic life support training on the learners' cardiopulmonary and automated external defibrillator skills. BioMed Research International. 2016; 2016: 2420568.

[16] Rössler B, Ziegler M, Hüpfl M, Fleischhackl R, Krychtiuk KA, Schebesta K. Can a flowchart improve the quality of bystander cardiopulmonary resuscitation? Resuscitation. 2013; 84: 982-986.

[17] Chung CH, Siu AYC, Po LLK, Lam CY, Wong PCY. Comparing the effectiveness of video self-instruction versus traditional classroom instruction targeted at cardiopulmonary resuscitation skills for laypersons: a prospective randomised controlled trial. Hong Kong Medical Journal. 2010; 16: 165-170.

[18] Baldi E, Cornara S, Contri E, Epis F, Fina D, Zelaschi B, et al. Realtime visual feedback during training improves laypersons' CPR quality: a randomized controlled manikin study. Canadian Journal of Emergency Medicine. 2017; 19: 480-487.

[19] Bobrow BJ, Vadeboncoeur TF, Spaite DW, Potts J, Denninghoff K, Chikani $\mathrm{V}$, et al. The effectiveness of ultrabrief and brief educational videos for training lay responders in hands-only cardiopulmonary resuscitation. Circulation: Cardiovascular Quality and Outcomes. 2011; 4: 220-226.

[20] Kim TH, Lee YJ, Lee EJ, Ro YS, Lee K, Lee H, et al. Comparison of cardiopulmonary resuscitation quality between standard versus telephone-basic life support training program in middle-aged and elderly housewives: a randomized simulation study. Simulation in Healthcare. 2018; 13: 27-32.

[21] Boet S, Bould MD, Pigford A, Rössler B, Nambyiah P, Li Q, et al. Retention of basic life support in laypeople: mastery learning vs. timebased education. Prehospital Emergency Care. 2017; 21: 362-377.

How to cite this article: Lourdes Luque-López, Jesús MolinaMula. Basic life support training for the adult lay population. A systematic review. Signa Vitae. 2021;17(3):47-61. doi:10.22514/sv.2021.026. 